\title{
Potential security factors in software cost estimation
}

\begin{abstract}
Software houses are now keen to provide secure software as requested by customersôdesire with respect to security and quality of their products especially related to the software costing estimation in the software development and implementation environment. Therefore, there is a need to identify the potential security risks while estimating the application cost. In this paper, we provide a list of potential security risks throughout the system development life cycle (SDLC). This list provides useful insights for software developers and practitioners in identifying security risks so that it can be encountered as security cost in the application.
\end{abstract}

